\title{
Effects of Solvent Polarity on Li-ion Diffusion in Polymer Electrolytes: An All-Atom Molecular Dynamics Study with Charge Scaling
}

\author{
Harish Gudla, Chao Zhang,* and Daniel Brandell
}

Cite This: J. Phys. Chem. B 2020, 124, 8124-8131

Read Online

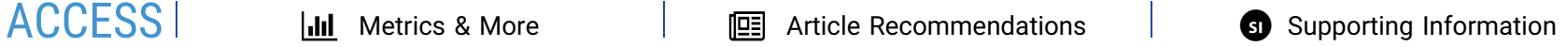

ABSTRACT: We herein report an all-atom molecular dynamics study on the role of solvent polarity for $\mathrm{Li}^{+}$diffusion in polymer electrolytes using PEO-LiTFSI (poly(ethylene oxide)-lithium bis(trifluoromethane)sulfonimide) as a model system. By separating the effect of $T_{\mathrm{g}}$ and the effect of solvent polarity in our simulations, we show that the maximum in the diffusion coefficient of $\mathrm{Li}^{+}$with respect to the dielectric constant of polymer solvent $\varepsilon_{\mathrm{p}}$ is due to transitions in the transport mechanism. In particular, it is found that the frequent interchain hopping involves the coordination of both PEO and TFSI. This optimal solvating

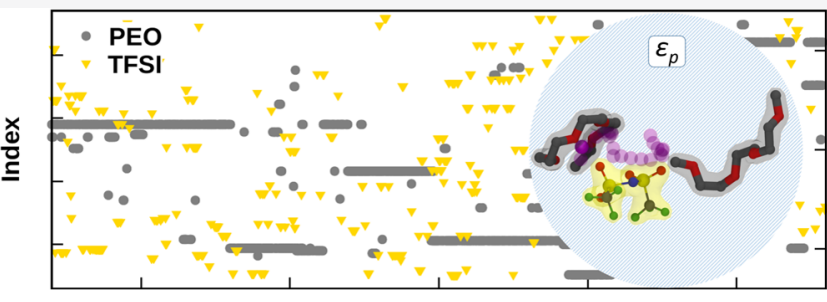

Time (ns) ability of PEO at an intermediate value of $\varepsilon_{\mathrm{p}}$ leads to the fast ion conduction. These findings highlight the synergetic effect of solvent polarity and bond polarity on Li-ion diffusion in solid polymer electrolytes.

\section{INTRODUCTION}

Solid (solvent-free) polymer electrolytes (SPEs) are promising alternatives to conventional liquid counterparts, which enhance the safety, the flexibility, and the reduction of the leakage in $\mathrm{Li}$ ion batteries. ${ }^{1,2}$ However, one of the remaining yet decisive bottlenecks in the development of polymer electrolytes is the limited ionic conductivity at room temperature. The ionic conductivity in polymer electrolytes depends on a variety of factors such as polymer structure, molecular weight, glass transition temperature $\left(T_{\mathrm{g}}\right)$, end/side-chain groups, salt concentration, donor number, solvent polarity, etc. ${ }^{3-10}$ To understand the coupling between these factors, to disentangle their contributions to the ion transportation, and to use this knowledge for designing new types of SPEs, constitute the grand challenges in the field. To this end, modeling by computer simulations can provide significant aid since these aspects can be studied systematically.

Factors such as solvent polarity, $T_{\mathrm{g}}$ and donor number control the solvating ability of salt in the polymer systems and thus directly affect the conductivity of ions. ${ }^{11-14}$ However, these factors are highly coupled, rendering it difficult to determine the influence of these parameters on ionic mobility and have been little explored for polymer electrolyte systems. In a recent experimental study on the ion conduction in poly(glycidyl ether)-based lithium bis(trifluoromethyl)sulfonimide (LiTFSI) electrolytes, ${ }^{15}$ it was found that the measured ionic conductivity (but not cation and anion diffusivities) increases monotonically with the host polymer dielectric constant. This triggered a full atomistic molecular dynamics (MD) simulations study on these electrolytes. ${ }^{16}$ The simulations could qualitatively reproduce the experimental trends and attributed the observed increase in ionic conductivity in the polymer matrix of high solvent polarity to an ameliorated ion aggregation and more free charge carriers. To further investigate the dependence of polymer polarity on ionic conduction, this study was followed by coarse-grained simulations using a Stockmayer model for neat and salt (LiTFSI) doped poly(ethylene oxide) (PEO) electrolytes. ${ }^{17}$ Therein, the point dipole moment of the EO monomer was scaled as an indicator of the solvent polarity strength. It was found that the ionic conductivity, as well as diffusivities from the Nernst-Einstein relation, reaches a maximum at an intermediate dipole strength. This optimal point was rationalized as a trade-off between ionic aggregation and polymer segmental dynamics, i.e., a further increased polarity reduces the polymer segmental motion due to enhanced polymer-polymer interactions and thereby reduces ion transport. However, the polarity of polymer electrolytes involves both the dielectric effect of the solvent and the bond polarity resulting from the local effect of coordination and quantified by the donor number. ${ }^{18}$ Moreover, the effect that the increased polymer dielectric constant leads to enhanced ionic conductivity may be solely attributed to the shift in $T_{\mathrm{g}}$, as

Received: June 5, 2020

Revised: August 24, 2020

Published: August 25, 2020 
shown in experiments by tuning the backbone polarity. ${ }^{19}$ Therefore, a fully atomistic picture beyond coarse-grained models for disentangling these factors is needed.

In all-atom simulations, many-body polarizable force field $^{20,21}$ can provide accurate results for electrolytes with $\mathrm{Li}$ salts through either the atomic polarizability model or the fluctuating charge model. However, these methods are limited by high computational cost for long time-scale simulations. Instead, scaling the point charges in force fields is an effective approach to introduce electronic polarizations where the point charges of salt ions are scaled down to screen the polymer-ion and ion-ion interactions. The charge scaling factor $(f)$ of salt ions is related to the optical dielectric constant of the solvent $\left(\epsilon_{\infty}\right)$ by $\mathrm{refs}^{22-25}$. In simulations of polymer electrolytes, a scaling factor between 0.5 and 0.8 has been commonly used for salt ions in recent years, ${ }^{26-29}$ which has shown to better reproduce experimental diffusivities. A less used approach in MD studies of SPEs is to scale the point charge of solvent molecules to modulate the solvent polarity. A well-known example of this type is the rigid three-point water model, which employs an enhanced dipole moment of $2.35 \mathrm{D}$ for liquid water simulations instead of the gas phase value of $1.85 \mathrm{D}^{30}$ Note that the effect of bond polarity is included implicitly by scaling charges of salt and solvent since the local solvation environment is dominated by the electrostatic interaction.

In this work, we aim to examine the effects of polarity by employing all-atom MD simulations with charge scaling and taking the PEO-LiTFSI system as a prototype. After describing the computational setups, we first show how to use the dielectric constant of polymer solvent $\varepsilon_{\mathrm{p}}$ to gauge the strength of solvent polarity and discuss the relation between solvent polarity and the glass transition temperature $\left(T_{\mathrm{g}}\right)$. Then, by separating the effect of $T_{\mathrm{g}}$ and the effect of solvent polarity, we show how solvent polarity affects polymer dynamics and ion conduction and leads to an optimal point in terms of the self-diffusion coefficient of $\mathrm{Li}^{+}$. This is followed by a discussion on how local solvation environments (bond polarity) affect the ion-transport mechanisms in polymer electrolyte systems and couples to the solvent polarity. Overall, these point out that the optimal solvating ability of PEO at the intermediate value of $\varepsilon_{\mathrm{p}}$ leads to the maximum of $D_{\mathrm{Li}^{+}}$and balanced interactions between $\mathrm{Li}$ ion with TSFI and PEO lead to frequent interchain hopping of $\mathrm{Li}^{+}$.

\section{COMPUTATIONAL METHODS}

MD simulation boxes comprising neat and LiTFSI-doped PEO systems were constructed, comprising 200 hydroxyl-terminated chains, each with 25 monomer units $(1.11 \mathrm{~kg} / \mathrm{mol})$ and $400 \mathrm{Li}$ and 400 TFSI ions, corresponding to a $\left[\mathrm{Li}^{+}\right] /[\mathrm{EO}]$ concentration ratio of 0.08 . The initial configurations of the simulation boxes were generated through a random arrangement of polymer chains and ions, using PACKMOL package. ${ }^{31}$ Then, the total energy of the initial system was minimized using the steepest descent algorithm.

General AMBER force field (GAFF) parameters ${ }^{32}$ were used for describing bonding and nonbonding interactions in PEO and LiTFSI. ${ }^{3-35}$ The AM1-BCC (bond charge correction) ${ }^{36}$ model was used to assign the partial atomic charges (see Section A in the Supporting Information), which emulates the $\mathrm{HF} / 6-31 \mathrm{G}^{*}$ electrostatic potential (ESP) of a molecule. The van der Waals parameters used were the same as those implemented in the standard AMBER force field. ${ }^{32}$
All MD simulations were performed using GROMACS 2018.1..$^{37,38}$ The long-range electrostatic interactions were employed through a particle mesh Ewald technique ${ }^{39}$ with an interpolation order of 4 and a grid size of $0.16 \mathrm{~nm}$. The shortrange cutoff distances of the van der Waals and Coulombic interaction in the direct space are $1 \mathrm{~nm}$. The bonds involving hydrogen atoms were constrained using the LINCS algorithm. ${ }^{40}$ For all MD simulations, the leapfrog integrator was employed with a time step of $1 \mathrm{fs}$.

MD simulations in the NVT (constant number, volume, and temperature) and NPT (constant number, pressure, and temperature) ensembles were performed subsequently for the purpose of equilibration using a Bussi-Donadio-Parrinello thermostat $^{41}$ and a Parrinello-Rahman barostat ${ }^{42}$ at $400 \mathrm{~K}$ and 1 bar for 5 and $10 \mathrm{~ns}$, respectively. In the NVT and NPT equilibration runs, the temperature and the density of the system were equilibrated respectively. During these simulations, the coupling constants for the thermostat and the barostat were set to $0.1 \mathrm{ps}$ and $2.0 \mathrm{ps}$, respectively. Appropriate polymer equilibration was confirmed by comparing the mean squared displacement (MSD) of the PEO chains at the end of simulation and $\mathrm{R}_{\mathrm{g}}{ }^{2}$ and calculating the polymer end-to-end vector autocorrelations (see Section B in the Supporting Information for details). To determine the $T_{\mathrm{g}}$ of both neat and salt-doped systems, a stepwise NPT cooling from 400 to $140 \mathrm{~K}$ with a step of $20 \mathrm{~K}$ was performed for $20 \mathrm{~ns}$ at each given temperature. The densities of the system at each temperature were calculated from the last $5 \mathrm{~ns}$ of the simulation. To investigate the structural and transport properties of the LiTFSI-doped PEO systems, NPT simulations were carried out for 300 to $600 \mathrm{~ns}$ at different temperatures.

In Table 1, each system is labeled according to the scaling factor $f$ on the point charges in a polymer $(\mathrm{P})$ and salt $(\mathrm{S})$. For example, in the system $\mathrm{P}_{1.50} \mathrm{~S}_{0.75}$, the charges on polymer and salt are scaled by factors of 1.50 and 0.75 respectively.

Table 1. System Notations for Different Charge Scaling Schemes on Polymer and Salt with Simulation Time and Box Size after Equilibrations and Calculated $T_{\mathrm{g}}$

$\begin{array}{lccccc}\begin{array}{c}\text { system } \\ \text { notation }\end{array} & \begin{array}{c}\text { charge } \\ \text { scaling factor } \\ \text { of PEO }\end{array} & \begin{array}{c}\text { charge } \\ \text { scaling } \\ \text { factor of salt }\end{array} & \begin{array}{c}\text { simulation } \\ \text { time }(\mathrm{ns})\end{array} & \begin{array}{c}\text { box } \\ \text { size } \\ \left(\mathrm{nm}^{3}\right)\end{array} & T_{\mathrm{g}}(\mathrm{K}) \\ \mathrm{P}_{1.50} \mathrm{~S}_{1.00} & 1.50 & 1.00 & 600 & 430 & 317(4) \\ \mathrm{P}_{1.20} \mathrm{~S}_{1.00} & 1.20 & & 400 & 437 & 308(2) \\ \mathrm{P}_{1.00} \mathrm{~S}_{1.00} & 1.00 & & 400 & 444 & 304(4) \\ \mathrm{P}_{0.75} \mathrm{~S}_{1.00} & 0.75 & & 550 & 452 & 295(4) \\ \mathrm{P}_{0.50} \mathrm{~S}_{1.00} & 0.50 & & 550 & 460 & 283(3) \\ \mathrm{P}_{1.50} \mathrm{~S}_{0.75} & 1.50 & 0.75 & 300 & 441 & 310(4) \\ \mathrm{P}_{1.33} \mathrm{~S}_{0.75} & 1.34 & & 300 & 443 & 304(4) \\ \mathrm{P}_{1.20} \mathrm{~S}_{0.75} & 1.20 & & 300 & 447 & 299(4) \\ \mathrm{P}_{1.00} \mathrm{~S}_{0.75} & 1.00 & & 300 & 450 & 294(5) \\ \mathrm{P}_{0.75} \mathrm{~S}_{0.75} & 0.75 & & 300 & 454 & 284(4) \\ \mathrm{P}_{0.56} \mathrm{~S}_{0.75} & 0.56 & & 300 & 457 & 277(2) \\ \mathrm{P}_{0.45} \mathrm{~S}_{0.75} & 0.45 & & 300 & 460 & 269(3) \\ \mathrm{P}_{1.50} & 1.50 & \text { Neat PEO } & & & \\ \mathrm{P}_{1.00} & 1.00 & & 50 & 325 & 302(3) \\ \mathrm{P}_{0.75} & 0.75 & & 50 & 347 & 286(2) \\ \mathrm{P}_{0.50} & 0.50 & & 50 & 351 & 280(3) \\ & & & 50 & 359 & 272(2)\end{array}$




\section{RESULTS AND DISCUSSION}

\subsection{Relation between Solvent Polarity and Glass} Transition Temperature. The dielectric constant of the polymer electrolyte $\varepsilon_{\mathrm{p}}$ reflects the strength of solvent polarity. ${ }^{43}$ Since the MD simulations here were performed with periodic boundary conditions and Ewald summation, the dielectric constant of neat and salt-doped PEO can be computed from the fluctuations in the total dipole moment $\boldsymbol{M}$ of the PEO via the equation for a nonpolarizable model ${ }^{44}$

$$
\varepsilon_{\mathrm{p}}=1+\frac{4 \pi}{3 \varepsilon_{0} V k_{\mathrm{B}} T}\left(\left\langle\mathbf{M}^{2}\right\rangle-\langle\mathbf{M}\rangle^{2}\right)
$$

where $\varepsilon_{0}$ is the vacuum permittivity, $k_{\mathrm{B}}$ is Boltzmann's constant, $T$ is the temperature, $V$ is the average volume of the simulation box, and \langle\rangle is the ensemble averages. In experimental studies, the dielectric constant can mainly be tuned by changing the polymer chemistry through side chains $^{15,16}$ or backbone. ${ }^{19}$ For the same polymer electrolyte, it can, for example, be varied also by changing the salt concentration and temperature, and then their relations to the ionic conductivity can be observed. ${ }^{45,46}$

The calculated $\varepsilon_{\mathrm{p}}$ value for neat $\mathrm{PEO}$ at $\mathrm{P}_{1.00}$ in Figure $1 \mathrm{a}$ is in good agreement with the reported experimental values in

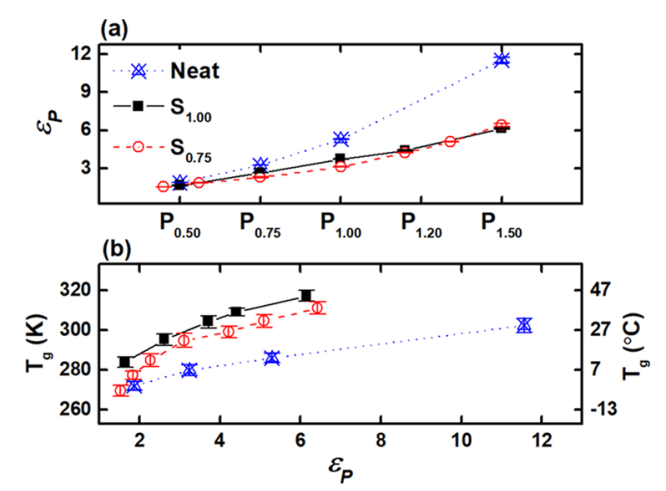

Figure 1. (a) Dielectric constants of PEO $\left(\varepsilon_{\mathrm{p}}\right)$ in the neat and saltdoped systems at different charge scaling schemes (see Table 1). (b) $T_{\mathrm{g}}$ of neat and salt-doped polymers as a function of the solvent polarity $\varepsilon_{\mathrm{p}}$.

the literature $(\varepsilon \sim 4-5) \cdot{ }^{16,47-49}$ Adding salt to the PEO matrix reduces the dielectric constant as the reorientation of the solvent is significantly hindered. From Figure 1a, one can see that the solvent polarity of a system was enhanced by scaling up the charge on the polymer. This dependence was expected, as the dipole moment is proportional to the atomic charge. The scaling of point charges of slat ions in the $S_{0.75}$ case will introduce an electronic screening in polymer-ion and ion-ion interactions. Interestingly, when comparing the results of $S_{1.00}$ and $S_{0.75}$ cases, it was found that the introduction of electronic polarization has a limited effect on the values of $\varepsilon_{\mathrm{p}}$. This confirms that the electronic polarization can be singled out by scaling charges of PEO and LiTFSI salt separately.

Before discussing the dependence of the glass transition temperature $T_{\mathrm{g}}$ on the solvent polarity, it is worth pointing out that the calculated $T_{\mathrm{g}}$ of $\mathrm{P}_{1.00} \mathrm{~S}_{1.00}$ is $304(4) \mathrm{K}$ (Table 1; also see Section $\mathrm{C}$ in the Supporting Information for details), which is about $75 \mathrm{~K}$ above the experimentally observed value of 230 $\mathrm{K}^{4,50}$ for the system with similar molecular weight $\left(M_{\mathrm{w}}=1.05\right.$ $\mathrm{kg} / \mathrm{mol}$ ) and salt concentration (0.05-0.1). This is commonly seen in MD simulations of SPEs due to limitations in the force field parameters. ${ }^{51}$ Because of this noticeable difference in the $T_{\mathrm{g}}$ between experimental and simulated systems, the normalized temperatures $\left(T-T_{0}\right)$ should be used. Here, $T_{0}$ is taken as $50 \mathrm{~K}$ below the $T_{\mathrm{g}}$, which was suggested to give the best fit to experimental data.

In Figure $1 \mathrm{~b}$, we can see that the $T_{\mathrm{g}}$ increases when the solvent polarity $\varepsilon_{\mathrm{p}}$ becomes larger. This is due to the increased polymer-polymer interactions that reduce the free volume. A comparison of the neat and LiTFSI-doped systems gives a difference of $10-20 \mathrm{~K}$ for all the cases, which is also observed experimentally. ${ }^{54,55}$ This originates from the restrictions of the polymer segmental motion with the inclusion of salt, which forms physical cross-links of the polymer chains. Since the electrostatic interactions are weaker between the salt and the polymer chains in the $S_{0.75}$ cases than those in the $S_{1.00}$ cases, this leads to the decrement of $T_{\mathrm{g}}$ in the system for $S_{0.75}$.

3.2. Impact of Solvent Polarity on Li-Ion Conduction and Polymer Dynamics. The self-diffusion coefficient $(D)$ for the ions and the polymer chains were used to probe polymer dynamics and ion mobility. $D$ is calculated from MSD curves using the Einstein relation

$$
D=\frac{1}{6 t} \sum_{i=1}^{N} \lim _{t \rightarrow \infty}\left\langle\left\|\mathbf{r}_{i}(t)-\mathbf{r}_{i}(0)\right\|^{2}\right\rangle
$$

where $\mathbf{r}_{i}(t)$ and $\mathbf{r}_{i}(0)$ are the center of the mass of entity $i$ at times $t$ and 0 , respectively. ${ }^{56}$ Specifically, the $\operatorname{MSD}(t)$ curves for $\mathrm{Li}^{+}$and TFSI were calculated using the center of mass of the corresponding ions, and those for polymer chains were obtained using the oxygen atoms. The self-diffusion coefficient was calculated using the diffusive regime of the simulation, i.e., $\operatorname{MSD}(t) \sim t$ (see Section D in the Supporting Information for details). In most cases, this was achieved after $\sim 150 \mathrm{~ns}$ at temperatures above $400 \mathrm{~K}$, while longer simulations (>500 ns) were needed at lower temperatures.

The self-diffusion coefficient of $\mathrm{Li}^{+}\left(D_{\mathrm{Li}^{+}}\right)$as a function of normalized temperature $\left(1000 /\left(T-T_{\mathrm{g}}+50\right)\right)$ is shown in Figure 2. It can be seen that when increasing the solvent

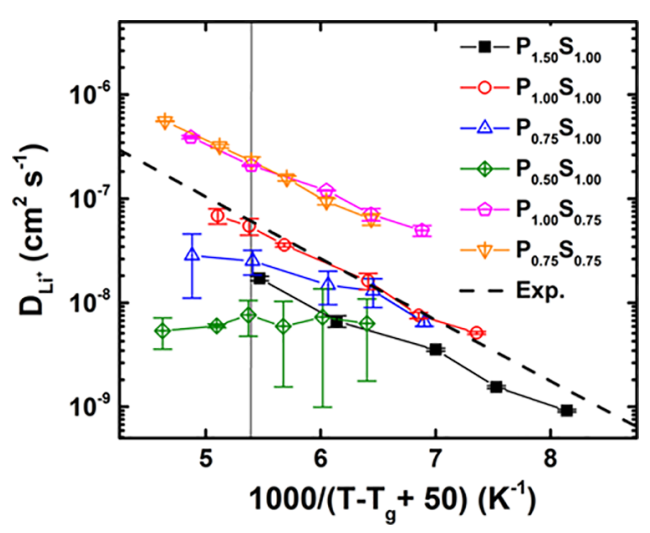

Figure 2. $\mathrm{Li}^{+}$self-diffusion coefficient $\left(D_{\mathrm{Li}^{+}}\right)$as a function of normalized inverse temperature for different charge scaling schemes. The experimental line was taken from ref 55 .

polarity from $\mathrm{P}_{0.50} \mathrm{~S}_{1.00}$ to $\mathrm{P}_{1.50} \mathrm{~S}_{1.00}$, the intercept of $D_{\mathrm{Li}^{+}}$vs $\left(1000 /\left(T-T_{\mathrm{g}}+50\right)\right)$ becomes smaller as well as the slope of the corresponding curve. This means that $\mathrm{Li}^{+}$diffusions in these polymer electrolyte systems follow the so-called MeyerNeldel rule in the low to intermediate range of $\varepsilon_{\mathrm{p}}$, where the prefactor goes down together with the activation energy. ${ }^{57} \mathrm{An}$ 
exception was found for the highest solvent polarity $\left(\mathrm{P}_{1.50}\right.$ $\left.\mathrm{S}_{1.00}\right)$, which suggests a different ion-transport mechanism, as will be discussed later.

When compared with the experimental data, ${ }^{55}$ the $\mathrm{P}_{1.00} \mathrm{~S}_{1.00}$ system seems to have the best agreement. However, this is fortuitous. Although the experimental PEO-LiTFSI system has the same salt concentration as the simulation system, the corresponding molecular weight is much higher $\left(M_{\mathrm{w}}=100 \mathrm{~kg} /\right.$ mol). In studies of the dependence of molecular weight and chain length of PEO on $D_{\mathrm{Li}^{+}}$, is has been shown that $D_{\mathrm{Li}^{+}}$ increases by $20-25 \%$ when $M_{\mathrm{w}}$ decreases from 100 to $1 \mathrm{~kg}$ / mol $^{5}$. This means that $S_{0.75}$, which includes the electronic polarization, might actually constitute a better approximation for use in MD simulations of this system.

To separate the different effects of solvent polarity and $T_{g}$, cross-sectional data for $D_{\mathrm{Li}^{+}}$at a chosen normalized inverse temperature of $1000 /\left(T-T_{\mathrm{g}}+50\right)=5.4( \pm 0.1)$, as indicated by the vertical line in Figure 2, were selected for a range of different polarities. As shown in Figure 3a, for both $S_{1.00}$ and
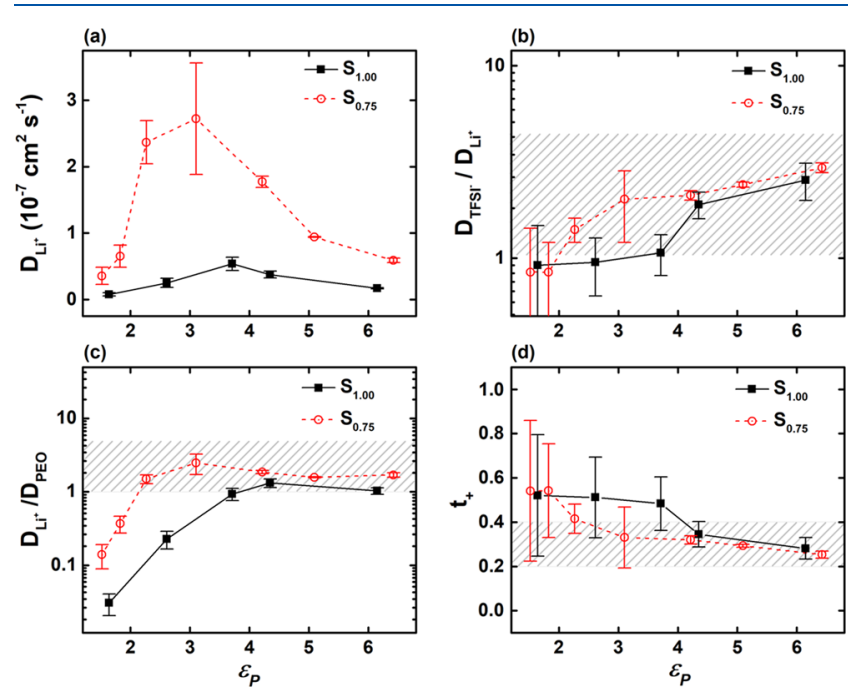

Figure 3. (a) $\mathrm{Li}^{+}$self-diffusion coefficient $\left(D_{\mathrm{Li}^{+}}\right),\left(\right.$b) $D_{\mathrm{TFSI}} / D_{\mathrm{Li}}{ }^{+}$, (c) $D_{\mathrm{Li}^{+}} / D_{\mathrm{PEO}}$, and (d) cation transference number $\left(t_{+}\right)$as a function of solvent polarity at the normalized inverse temperature $1000 /\left(T-T_{\mathrm{g}}\right.$ $+50)$ of $5.4( \pm 0.1)$. Gray region corresponds to the expected values $D_{\mathrm{TFSI}} / D_{\mathrm{Li}^{+}}>1, D_{\mathrm{Li}}^{+} / D_{\mathrm{PEO}}>1$ and $t_{+} \sim 0.2-0.4$.

$\mathrm{S}_{0.75}$ cases, similar trends are found and maxima in $D_{\mathrm{Li}^{+}}\left(\varepsilon_{\mathrm{p}}\right)$ appear at $\varepsilon_{\mathrm{p}}=3-4$. However, the maximum is shifted to lower $\varepsilon_{\mathrm{p}}$ and a higher $D_{\mathrm{Li}^{+}}$is obtained in the case of $\mathrm{S}_{0.75}$. These observations will be discussed later by looking at the iontransport mechanisms and local coordination environments (Sections 3.3 and 3.4).

In the PEO-LiTFSI systems, it is known from experiments that the magnitude of self-diffusion coefficients of each component should follow the order $D_{\mathrm{TFSI}} \gg D_{\mathrm{Li}^{+}}>D_{\mathrm{PEO}}$ during extended time scales. ${ }^{58,59}$ In Figure $3 b, c$, one can see how the solvent polarity modulates this sequence. It is found that when the value of $\varepsilon_{\mathrm{p}}$ is low, $D_{\mathrm{PEO}} \gg D_{\mathrm{TFSI}} \sim D_{\mathrm{Li}^{+}}$. This suggests that there is significant ion-pairing due to the poor screening from the solvent and that the diffusion of $\mathrm{Li}^{+}$is decoupled from the polymer matrix. Instead, when $\varepsilon_{\mathrm{p}}$ becomes larger, the expected order $D_{\mathrm{TFSI}} \gg D_{\mathrm{Li}^{+}}>D_{\mathrm{PEO}}$ appears. In this regime, $D_{\mathrm{Li}^{+}} / D_{\mathrm{PEO}}$ is larger than 1 , which indicates that the lithium-ion transport occurs via a hopping mechanism between different coordinating sites.
In addition, the cation transference number $\left(t_{+}\right)$was calculated from self-diffusion coefficients of cation and anion as follows

$$
t_{+}=\frac{D_{\mathrm{Li}^{+}}}{D_{\mathrm{Li}^{+}}+D_{\mathrm{TFSI}}}
$$

Figure $3 \mathrm{~d}$ shows the dependence of $t_{+}$on solvent polarity. The $t_{+}$is nearly 0.5 at low $\varepsilon_{\mathrm{p}}$ values, which is likely coupled to the formation of ion pairs and that the ionic species migrate together. At high $\varepsilon_{\mathrm{p}}$, on the other hand, the $t_{+}$values are closer to experimentally observed transference numbers; $0.2-0.3$ for systems with a similar molecular weight of polymer at the same salt concentration. ${ }^{4,6,59}$

3.3. Impact of Solvent Polarity on Li-Ion Transport Mechanism. As discussed, the effect of solvent polarity on Liion mobility is not monotonic. For example, the case of $\mathrm{P}_{1.50} \mathrm{~S}_{1.00}$ does not follow the Meyer-Neldel rule (Figure 2). In this case, the ratio $D_{\mathrm{Li}^{+}} / D_{\text {PEO }}$ goes from below 1 to above 1 (Figure $3 \mathrm{c}$ ), and the maximum $D_{\mathrm{Li}^{+}}$value in the $\mathrm{S}_{0.75}$ case is shifted toward lower $\varepsilon_{\mathrm{p}}$ and significantly larger than those in the case of $S_{1.00}$. All these observations point in the same direction: the Li-ion transport mechanisms are not the same when varying $\varepsilon_{\mathrm{p}}$.

To investigate the $\mathrm{Li}^{+}$transport mechanism, the local solvation environment in the simulation box was tracked for the entire trajectories of the ions during the sampling time. The first maximum of the radial distribution function (RDF) between $\mathrm{Li}-\mathrm{O}(\mathrm{PEO})$ was considered as the cutoff distance (see Supporting Information Section F, Figure S5). The indices of the PEO chains $(1-200)$ within this cutoff distance were recorded every $200 \mathrm{ps}$, which is much shorter than the residence time of $\mathrm{Li}^{+}$(see Section E in the Supporting Information for details). Typical representative time evolutions of the $\mathrm{Li}^{+}$coordination to these PEO chain indices for systems with high, intermediate, and low solvent polarities are presented in Figure $4 \mathrm{a}, \mathrm{c}, \mathrm{d}$ for the $\mathrm{S}_{1.00}$ case and in Figure $4 \mathrm{~b}, \mathrm{~d}, \mathrm{f}$ for the $\mathrm{S}_{0.75}$ case for selected individual $\mathrm{Li}^{+}$ions in these different systems.

It can be seen from Figure $4 \mathrm{a}, \mathrm{b}$ that at high $\varepsilon_{\mathrm{p}}, \mathrm{Li}^{+}$ions typically stay with one or two PEO chains for more than 100 ns and then hop to another chain. This shows that the transport mechanism, in this case, was mainly intrachain diffusion, either as hopping between different oxygens on the same chain or as diffusion together with the polymer. At intermediate $\varepsilon_{\mathrm{p}}$, where $D_{\mathrm{Li}^{+}}$is maximum, the interchain hopping between different PEO molecules becomes much more frequent (Figure $4 \mathrm{c}, \mathrm{d}$ ). At low $\varepsilon_{\mathrm{p}}$, the $\mathrm{Li}^{+}$ions seem to be barely coordinated to the PEO chains, which suggests they are instead mostly coordinated to TFSI ions (Figure 4e,f).

To confirm that the picture given by Figure $4 a-f$ is indeed statistically significant, the probability of the number of PEO chains visited by $\mathrm{Li}^{+}$was calculated, and the corresponding distributions are presented in Figure 4g,h. It is seen that with an increase in solvent polarity, the average number of PEO chains visited by the individual $\mathrm{Li}^{+}$ions increase initially and then decreases at high $\varepsilon_{\mathrm{p}}$. This is in accordance with Figure $4 \mathrm{a}-\mathrm{f}$ and suggests a transition of the transport mechanism from a vehicular mechanism at low $\varepsilon_{\mathrm{p}}$, to the frequent interchain hopping at intermediate $\varepsilon_{\mathrm{p}}$, and to the intrachain hopping at high $\varepsilon_{\mathrm{p}}$.

Previous studies ${ }^{58,60-62}$ using a similar procedure have shown that the $\mathrm{Li}$ ions transported via interchain hopping 

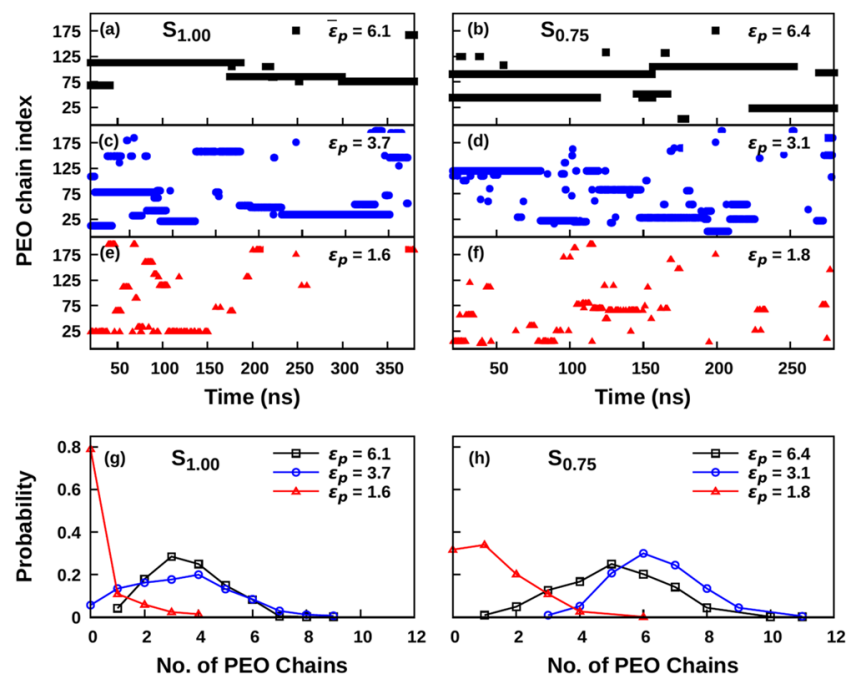

Figure 4. $\mathrm{Li}^{+}$-PEO chain coordination environments as a function of simulation time for the $S_{1.00}$ cases at $\varepsilon_{\mathrm{p}}=6.1(\mathrm{a}), 3.7(\mathrm{c})$, and 1.6 (e) and for the $S_{0.75}$ cases at $\varepsilon_{\mathrm{p}}=6.4(\mathrm{~b}), 3.1$ (d), and $1.8(\mathrm{f})$. The probability of the number of $\mathrm{PEO}$ chains visited by $\mathrm{Li}^{+}$ion for the $\mathrm{S}_{1.00}(\mathrm{~g})$ and $\mathrm{S}_{0.75}(\mathrm{~h})$ cases. One visit is counted if $\mathrm{Li}^{+}$stays with that PEO chain for more than $5 \%$ of simulation time $(\sim 20-30 \mathrm{~ns})$.

events have a higher diffusion coefficient than that from intrachain hopping or vehicular mechanisms. In fact, the vehicular mobility only corresponds to transport on a very short time scale, since the global macromolecular diffusivity at longer time scales is negligible, at least for higher molecular weights where the polymers are entangled. Consequently, this suggests that more frequent interchain hopping would lead to the highest diffusion coefficients, thereby justifying the maximum $D_{\mathrm{Li}^{+}}$found in Figure 3a.

Previously, it has been shown that a further increase in polarity reduces the polymer segmental motion and leads to slow Li-ion diffusion. ${ }^{17}$ This agrees with the observations in Figure $4 \mathrm{a}, \mathrm{b}$. However, characterizing the solvent polarity solely by $\varepsilon_{\mathrm{p}}$ should be treated with caution. As mentioned in the Introduction, charge scaling does not only modulate $\varepsilon_{\mathrm{p}}$ explicitly but also changes the bond polarity implicitly. Therefore, further analysis on the local Li-ion solvation environments during Li-on conduction is necessary to shed light on this issue.

3.4. Importance of the Local Solvation Environment on Li-Ion Conduction. The local solvation environment of $\mathrm{Li}^{+}$in the system is studied from its coordination number (CN). The CN of oxygen atoms in TFS and in the PEO chains around $\mathrm{Li}^{+}$can be calculated by integrating their RDFs, $g(r)$, to its first minimum $\left(r_{\text {min }}\right)$, thereby only including the first coordination shell

$$
\mathrm{CN}_{\mathrm{Li}^{+}-\mathrm{O}(\mathrm{X})}\left(r_{\min }\right)=4 \pi \rho \int_{0}^{r_{\min }} g_{\mathrm{Li}^{+}-\mathrm{O}(\mathrm{X})}(r) r^{2} \mathrm{~d} r
$$

where $\rho$ is the number density of oxygen atoms. The average number of $\mathrm{O}$ atoms around $\mathrm{Li}^{+}$in the first coordination shell for all systems is $5.3( \pm 0.6)$. These observations are in good agreement with NDIS (neutron diffraction with isotopic substitution) experiments ${ }^{63}$ and MD simulations ${ }^{33,58,59}$ of similar systems.

The calculated $\mathrm{CNs}$ for $\mathrm{Li}^{+}-\mathrm{O}(\mathrm{PEO})$ and $\mathrm{Li}^{+}-\mathrm{O}$ (TFSI) in different polarization environments are plotted in Figure 5. At lower solvent polarity, $\mathrm{Li}^{+}$is mostly coordinated by TFSI for

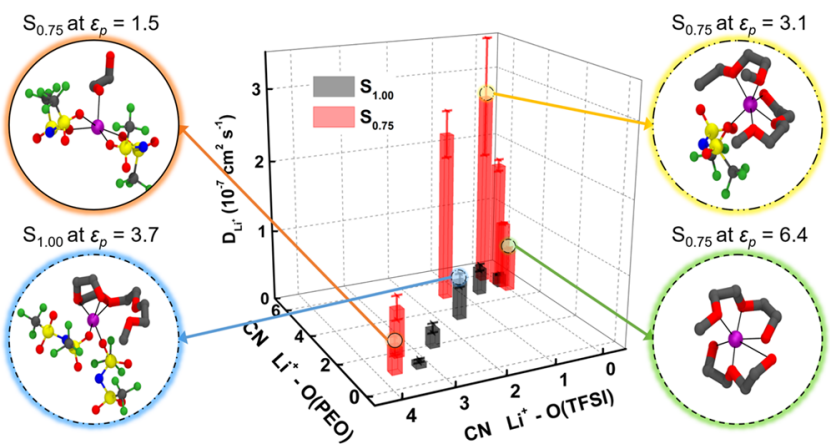

Figure 5. Three-dimensional (3D) bar plot of $D_{\mathrm{Li}^{+}}$as functions of coordination numbers (CNs) of $\mathrm{Li}^{+}-\mathrm{O}(\mathrm{PEO})$ (bottom left axis) and $\mathrm{Li}^{+}-\mathrm{O}$ (TFSI) (bottom right axis) at the normalized inverse temperature $1000 /\left(T-T_{\mathrm{g}}+50\right)$ of $5.4( \pm 0.1)$. Representative snapshots of the first coordination shell of $\mathrm{Li}^{+}$in different polarization environments are also shown. These snapshots show the crossover in $\mathrm{Li}^{+}$ coordination environment from TFSI to PEO with an increase in solvent polarity. Color coding of atoms: $\mathrm{Li}$, violet; $\mathrm{C}$, gray; $\mathrm{N}$, blue; $\mathrm{O}$, red; F, green; and S, yellow.

both $S_{1.00}$ and $S_{0.75}$ cases (also see the snapshot on the top left side in Figure 5). This agrees with the 0.5 transference number found at low solvent polarity (Figure $3 \mathrm{~d}$ ), signaling that the ions have similar diffusivities and therefore are moving together. As the solvent polarity increases, there is a crossover in $\mathrm{CNs}$ of $\mathrm{Li}^{+}-\mathrm{O}(\mathrm{PEO})$ and $\mathrm{Li}^{+}-\mathrm{O}(\mathrm{TFSI})$ for both $\mathrm{S}_{1.00}$ and $\mathrm{S}_{0.75}$ (which were also illustrated in Figure 5). The $\mathrm{CN}$ of $\mathrm{Li}^{+}-$ $\mathrm{O}$ (TFSI) is negligible at high solvent polarity, which indicates $\mathrm{Li}^{+}$is mostly coordinated by the polymer matrix (the snapshot on the bottom right in Figure 5). To further illustrate the solvent environments, the fraction of contact ion pairs (CIPs) and the solvent separated ion pairs (SSIPs) were also calculated using cutoff distances from Li-O(TFSI) radial distribution functions (see Section $F$ in the Supporting Information for details). From Figure S6, for both $S_{1.00}$ and $S_{0.75}$ cases, a crossover from CIPs to SSIPs can be observed at the solvent polarity where the maximum in $\mathrm{D}_{\mathrm{Li}^{+}}{ }^{+}$is observed. This crossover point is in good agreement with the crossover point found for the CN of $\mathrm{Li}-\mathrm{O}(\mathrm{PEO})$ and $\mathrm{Li}-\mathrm{O}$ (TFSI).

What is important here is that the maximum $D_{\mathrm{Li}^{+}}$shows up when the coordination of Li-ion involves both PEO and TFSI (the snapshot on the bottom left and top right in Figure 5). This suggests that an optimal solvating ability of polymer functional groups is crucial for fast Li-ion conduction in SPEs.

For systems having the maximum $D_{\mathrm{Li}^{+}}$, it is found that TFSI facilitates the interchain hopping among the PEO chains. The $\mathrm{Li}^{+}$-PEO chain and $\mathrm{Li}^{+}$-TFSI coordination environments as a function of simulation time during the interchain hopping is given in Figure 6. In both $S_{1.00}$ and $S_{0.75}$ cases, the TFSI ions clearly get involved in the process. The TFSI ions thereby participate in forming good structural connectivity sites for ion hopping, which is necessary for fast ion conduction. ${ }^{64}$ However, the $\mathrm{Li}^{+}$is mostly coordinated to the same TFSI ions in the case of $S_{1.00}$ whereas it hops between different TFSI ions in the case of $S_{0.75}$. This difference may be attributed to the effect of electronic polarization through charge scaling of the salt.

\section{CONCLUSIONS}

In this work, the effect of the solvent polarity of PEO electrolytes on the $\mathrm{Li}^{+}$diffusion has been investigated by 

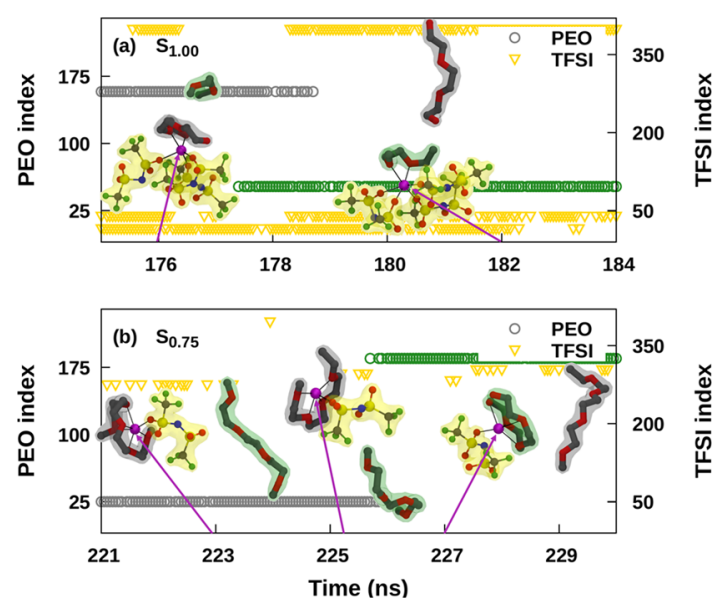

Figure 6. $\mathrm{Li}^{+}$-PEO chain and $\mathrm{Li}^{+}$-TFSI coordination environments as a function of simulation time during the interchain hopping (gray to green PEO chain) for the $S_{1.00}$ case at $\varepsilon_{\mathrm{p}}=3.7$ (a) and for the $S_{0.75}$ case at $\varepsilon_{\mathrm{p}}=3.1(\mathrm{~b})$. Color coding of atoms: $\mathrm{Li}$, violet; C, gray; $\mathrm{N}$, blue; $\mathrm{O}$, red; $\mathrm{F}$, green; and $\mathrm{S}$, yellow. Color coding of surfaces: $\mathrm{PEO}$, gray and green, and TFSI, yellow.

employing charge scaling on PEO chains. All-atom MD simulations were carried to elucidate the coupling between local solvation environments and solvent polarity, which is out of reach for coarse-grained simulations. By comparing two cases where the point charges of LiTFSI are either intact or scaled down, both ionic and electronic screening effects of solvent were taken into account. It is found that the $T_{\mathrm{g}}$ of the PEO-LiTFSI system becomes higher with the increment of the solvent polarity as characterized by the dielectric constant of PEO, i.e., $\varepsilon_{\mathrm{p}}$. The dependence of $D_{\mathrm{Li}^{+}}$on the normalized temperature follows the so-called Meyer-Neldel rule in the low to intermediate range of $\varepsilon_{\mathrm{p}}$.

By choosing a certain temperature normalized to $T_{\mathrm{g}}$, the effect of $T_{\mathrm{g}}$ and the effect of solvent polarity were separated in our analysis. It was found that the maximum in the diffusion coefficient of $\mathrm{Li}^{+}$appears for intermediate values of $\varepsilon_{\mathrm{p}}$. This is related to the fact that the ratio $D_{\mathrm{Li}+} / D_{\mathrm{PEO}}$ goes from below 1 to above 1 with increasing $\varepsilon_{\mathrm{p}}$. The corresponding transport mechanism changes from the vehicular mechanism, to the frequent interchain hopping, and then to the intrachain hopping. Since charge scaling does not only modulate $\varepsilon_{\mathrm{p}}$ explicitly but also implicitly tweaks the bond polarity (thereby the donor number), the maximum of $D_{\mathrm{Li}^{+}}$can be rationalized by the optimal solvating ability of PEO at the intermediate value of $\varepsilon_{\mathrm{p}}$. In particular, it was seen that balanced interactions between Li-ion with TSFI and PEO led to the frequent interchain hopping and the fast ion conduction.

These findings reveal the synergetic effect between solvent polarity and bond polarity for fast Li-ion diffusion in polymer electrolytes and provide insight for the rational design of the novel polymer electrolytes for solid-state Li-ion batteries. ${ }^{65,66}$

\section{ASSOCIATED CONTENT}

\section{SI Supporting Information}

The Supporting Information is available free of charge at https://pubs.acs.org/doi/10.1021/acs.jpcb.0c05108.

Initial partial charges from the force field; equilibration checks for polymers in the system; determination of glass transition temperature $\left(T_{\mathrm{g}}\right)$, self-diffusion coef- ficients from $\mathrm{MSD}, \mathrm{Li}^{+}-\mathrm{O}(\mathrm{PEO})$ residence time, radial distribution functions, and ion-pairing fractions (PDF)

\section{AUTHOR INFORMATION}

\section{Corresponding Author}

Chao Zhang - Department of Chemistry-Ångström

Laboratory, Uppsala University, 75121 Uppsala, Sweden; (1) orcid.org/0000-0002-7167-0840; Email: chao.zhang@

kemi.uu.se

\section{Authors}

Harish Gudla - Department of Chemistry-Ångström Laboratory, Uppsala University, 75121 Uppsala, Sweden

Daniel Brandell - Department of Chemistry-Ångström Laboratory, Uppsala University, 75121 Uppsala, Sweden; (1) orcid.org/0000-0002-8019-2801

Complete contact information is available at:

https://pubs.acs.org/10.1021/acs.jpcb.0c05108

\section{Notes}

The authors declare no competing financial interest.

\section{ACKNOWLEDGMENTS}

This work has been supported by the European Research Council (ERC), grant no. 771777 "FUN POLYSTORE". C.Z. thanks funding from the Swedish National Strategic e-Science program eSSENCE. The simulations were performed on the resources provided by the Swedish National Infrastructure for Computing (SNIC) at NSC and PDC. We thank J. Mindemark for helpful discussions.

\section{REFERENCES}

(1) Xue, Z.; He, D.; Xie, X. Poly(Ethylene Oxide)-Based Electrolytes for Lithium-Ion Batteries. J. Mater. Chem. A 2015, 3, 19218-19253.

(2) Li, Q.; Chen, J.; Fan, L.; Kong, X.; Lu, Y. Progress in Electrolytes for Rechargeable Li-Based Batteries and Beyond. Green Energy Environ. 2016, 1, 18-42.

(3) Teran, A. A.; Tang, M. H.; Mullin, S. A.; Balsara, N. P. Effect of Molecular Weight on Conductivity of Polymer Electrolytes. Solid State Ionics 2011, 203, 18-21.

(4) Devaux, D.; Bouchet, R.; Glé, D.; Denoyel, R. Mechanism of Ion Transport in PEO/LiTFSI Complexes: Effect of Temperature, Molecular Weight and End Groups. Solid State Ionics 2012, 227, 119-127.

(5) Timachova, K.; Watanabe, H.; Balsara, N. P. Effect of Molecular Weight and Salt Concentration on Ion Transport and the Transference Number in Polymer Electrolytes. Macromolecules 2015, 48, $7882-7888$.

(6) Pożyczka, K.; Marzantowicz, M.; Dygas, J. R.; Krok, F. Ionic Conductivity and Lithium Transference Number of Poly(Ethylene Oxide):LiTFSI System. Electrochim. Acta 2017, 227, 127-135.

(7) Brandell, D.; Priimägi, P.; Kasemägi, H.; Aabloo, A. Branched Polyethylene/Poly(Ethylene Oxide) as a Host Matrix for Li-Ion Battery Electrolytes: A Molecular Dynamics Study. Electrochim. Acta 2011, 57, 228-236.

(8) Sengwa, R. J.; Kaur, K.; Chaudhary, R. Dielectric Properties of Low Molecular Weight Poly(Ethylene Glycol)s. Polym. Int. 2000, 49, 599-608.

(9) Karmakar, A.; Ghosh, A. Dielectric Permittivity and Electric Modulus of Polyethylene Oxide (PEO)-LiClO4 Composite Electrolytes. Curr. Appl. Phys. 2012, 12, 539-543.

(10) Kim, C. S.; Oh, S. M. Importance of Donor Number in Determining Solvating Ability of Polymers and Transport Properties in Gel-Type Polymer Electrolytes. Electrochim. Acta 2000, 45, 21012109. 
(11) Ketkar, P. M.; Shen, K. H.; Hall, L. M.; Epps, T. H. Charging toward Improved Lithium-Ion Polymer Electrolytes: Exploiting Synergistic Experimental and Computational Approaches to Facilitate Materials Design. Mol. Syst. Des. Eng. 2019, 4, 223-238.

(12) Shen, K. H.; Hall, L. M. Ion Conductivity and Correlations in Model Salt-Doped Polymers: Effects of Interaction Strength and Concentration. Macromolecules 2020, 10, 58.

(13) Nakamura, I.; Wang, Z. G. Effects of Dielectric Inhomogeneity in Polyelectrolyte Solutions. Soft Matter 2013, 9, 5686-5690.

(14) Lin, K. J.; Maranas, J. K. Cation Coordination and Motion in a Poly(Ethylene Oxide)-Based Single Ion Conductor. Macromolecules 2012, 45, 6230-6240.

(15) Barteau, K. P. Poly(Glycidyl Ether)-Based Battery Electrolytes: Correlating Polymer Properties to Ion Transport. Ph.D. Thesis, University of California: Santa Barbara, CA, 2015.

(16) Wheatle, B. K.; Keith, J. R.; Mogurampelly, S.; Lynd, N. A.; Ganesan, V. Influence of Dielectric Constant on Ionic Transport in Polyether-Based Electrolytes. ACS Macro Lett. 2017, 6, 1362-1367.

(17) Wheatle, B. K.; Lynd, N. A.; Ganesan, V. Effect of Polymer Polarity on Ion Transport: A Competition between Ion Aggregation and Polymer Segmental Dynamics. ACS Macro Lett. 2018, 7, 11491154.

(18) Gutmann, V. Solvent Effects on the Reactivities of Organometallic Compounds. Coord. Chem. Rev. 1976, 18, 225-255.

(19) Schauser, N. S.; Grzetic, D. J.; Tabassum, T.; Kliegle, G. A.; Le, M. L.; Susca, E. M.; Antoine, S.; Keller, T. J.; Delaney, K. T.; Han, S.; et al. The Role of Backbone Polarity on Aggregation and Conduction of Ions in Polymer Electrolytes. J. Am. Chem. Soc. 2020, 142, 70557065.

(20) Borodin, O.; Smith, G. D. Development of Many-Body Polarizable Force Fields for Li-Battery Components: 1. Ether, Alkane, and Carbonate-Based Solvents. J. Phys. Chem. B 2006, 110, 62796292.

(21) Bedrov, D.; Piquemal, J. P.; Borodin, O.; MacKerell, A. D.; Roux, B.; Schröder, C. Molecular Dynamics Simulations of Ionic Liquids and Electrolytes Using Polarizable Force Fields. Chem. Rev. 2019, 119, 7940-7995.

(22) De Leeuw, S. W.; van Zon, A.; Bel, G. J. Structural Relaxation in Poly(Ethyleneoxide) and Poly(Ethyleneoxide)-Sodium Iodide Systems: A Molecular Dynamics Study. Electrochim. Acta 2001, 46, $1419-1426$.

(23) Leontyev, I. V.; Stuchebrukhov, A. A. Electronic Continuum Model for Molecular Dynamics Simulations. J. Chem. Phys. 2009, 130, 85102-85109.

(24) Pegado, L.; Marsalek, O.; Jungwirth, P.; Wernersson, E. Solvation and Ion-Pairing Properties of the Aqueous Sulfate Anion: Explicit versus Effective Electronic Polarization. Phys. Chem. Chem. Phys. 2012, 14, 10210-10248.

(25) Kirby, B. J.; Pavel, J. Charge Scaling Manifesto: A Way of Reconciling the Inherently Macroscopic and Microscopic Natures of Molecular Simulations. J. Phys. Chem. Lett. 2019, 10, 7531-7536.

(26) Chen, X.; Chen, F.; Liu, M. S.; Forsyth, M. Polymer Architecture Effect on Sodium Ion Transport in PSTFSI-Based Ionomers: A Molecular Dynamics Study. Solid State Ionics 2016, 288, 271-276.

(27) Molinari, N.; Mailoa, J. P.; Kozinsky, B. Effect of Salt Concentration on Ion Clustering and Transport in Polymer Solid Electrolytes: A Molecular Dynamics Study of PEO-LiTFSI. Chem. Mater. 2018, 30, 6298-6306.

(28) Mogurampelly, S.; Ganesan, V. Structure and Mechanisms Underlying Ion Transport in Ternary Polymer Electrolytes Containing Ionic Liquids. J. Chem. Phys. 2017, 146, No. 074902.

(29) Costa, L. T.; Sun, B.; Jeschull, F.; Brandell, D. Polymer-Ionic Liquid Ternary Systems for Li-Battery Electrolytes: Molecular Dynamics Studies of LiTFSI in a EMIm-TFSI and PEO Blend. J. Chem. Phys. 2015, 143, No. 024904.

(30) Jorgensen, W. L.; Chandrasekhar, J.; Madura, J. D.; Impey, R. W.; Klein, M. L. Comparison of Simple Potential Functions for Simulating Liquid Water. J. Chem. Phys. 1983, 79, 926.
(31) Martínez, L.; Andrade, R.; Birgin, E. G.; Martínez, J. M. Packmol: A Package for Building Initial Configurations for Molecular Dynamics Simulations. J. Comput. Chem. 2009, 30, 2157-2164.

(32) Wang, J.; Wolf, R. M.; Caldwell, J. W.; Kollman, P. A.; Case, D. A. Development and Testing of a General Amber Force Field. J. Comput. Chem. 2004, 25, 1157-1174.

(33) Ebadi, M.; Costa, L. T.; Araujo, C. M.; Brandell, D. Modelling the Polymer Electrolyte/Li-Metal Interface by Molecular Dynamics Simulations. Electrochim. Acta 2017, 234, 43-51.

(34) Sprenger, K. G.; Jaeger, V. W.; Pfaendtner, J. The General AMBER Force Field (GAFF) Can Accurately Predict Thermodynamic and Transport Properties of Many Ionic Liquids. J. Phys. Chem. B 2015, 119, 5882-5895.

(35) Liu, H.; Paddison, S. J. Alkyl Chain Length Dependence of Backbone-to-Backbone Distance in Polymerized Ionic Liquids: An Atomistic Simulation Perspective on Scattering. Macromolecules 2017, 50, 2889-2895.

(36) Jakalian, A.; Jack, D. B.; Bayly, C. I. Fast, Efficient Generation of High-Quality Atomic Charges. AM1-BCC Model: II. Parameterization and Validation. J. Comput. Chem. 2002, 23, 1623-1641.

(37) Abraham, M. J.; van der Spoel, D.; Lindah, E.; Hess, B. The GROMACS Development Team, GROMACS User Manual version, 2018. http://www.gromacs.org.

(38) Abraham, M. J.; Murtola, T.; Schulz, R.; Páll, S.; Smith, J. C.; Hess, B.; Lindah, E. GROMACS: High Performance Molecular Simulations through Multi-Level Parallelism from Laptops to Supercomputers. SoftwareX 2015, 1-2, 19-25.

(39) Darden, T.; York, D.; Pedersen, L. Particle Mesh Ewald: An N. $\log (\mathrm{N})$ Method for Ewald Sums in Large Systems. J. Chem. Phys. 1993, 98, 10089-10092.

(40) Hess, B.; Bekker, H.; Berendsen, H. J. C.; Fraaije, J. G. E. M. LINCS: A Linear Constraint Solver for Molecular Simulations. J. Comput. Chem. 1997, 18, 1463-1472.

(41) Bussi, G.; Donadio, D.; Parrinello, M. Canonical Sampling through Velocity Rescaling. J. Chem. Phys. 2007, 126, No. 014101.

(42) Parrinello, M.; Rahman, A. Polymorphic Transitions in Single Crystals: A New Molecular Dynamics Method. J. Appl. Phys. 1981, 52, $7182-7190$.

(43) Griffiths, T. R.; Pugh, D. C. Correlations among Solvent Polarity Scales, Dielectric Constant and Dipole Moment, and a Means to Reliable Predictions of Polarity Scale Values from Current Data. Coord. Chem. Rev. 1979, 29, 129-211.

(44) Neumann, M. Dipole Moment Fluctuation Formulas in Computer Simulations of Polar Systems. Mol. Phys. 1983, 50, 841858.

(45) Aziz, S. B.; Abidin, Z. H. Z. Electrical Conduction Mechanism in Solid Polymer Electrolytes: New Concepts to Arrhenius Equation. J. Soft Matter 2013, 2013, 1-8.

(46) Aziz, S. B. Li+ Ion Conduction Mechanism in Poly ( $\varepsilon$ Caprolactone)-Based Polymer Electrolyte. Iran. Polym. J. 2013, 22, $877-883$.

(47) Hanke, E.; Von Roden, K.; Kaatze, U. Hydrogen Network Fluctuations of Associating Liquids: Dielectric Relaxation of Ethylene Glycol Oligomers and Their Mixtures with Water. J. Chem. Phys. 2006, $125,84507$.

(48) Kammer, H. W. Dielectric Relaxation in PEO-Based Polymer Electrolytes. Ionics 2018, 24, 1415-1428.

(49) Matsumiya, Y.; Balsara, N. P.; Kerr, J. B.; Inoue, T.; Watanabe, H. In Situ Dielectric Characterization of Poly(Ethylene Oxide) Melts Containing Lithium Perchlorate under Steady Shear Flow. Macromolecules 2004, 37, 544-553.

(50) Hayamizu, K.; Akiba, E.; Bando, T.; Aihara, Y. 1H, 7Li, and 19F Nuclear Magnetic Resonance and Ionic Conductivity Studies for Liquid Electrolytes Composed of Glymes and Polyethene glycol Dimethyl Ethers of $\mathrm{CH} 3 \mathrm{O}(\mathrm{CH} 2 \mathrm{CH} 2 \mathrm{O}) \mathrm{NCH} 3(\mathrm{n}=3-50)$ Doped with $\mathrm{LiN}(\mathrm{SO} 2 \mathrm{CF} 3) 2$. J. Chem. Phys. 2002, 117, 5929-5939.

(51) Wu, C. Simulated Glass Transition of Poly(Ethylene Oxide) Bulk and Film: A Comparative Study. J. Phys. Chem. B 2011, 115, 11044-11052. 
(52) Lascaud, S.; Perrier, M.; Vallée, A.; Besner, S.; Prud'homme, J.; Armand, M. Phase Diagrams and Conductivity Behavior of Poly(Ethylene Oxide)-Molten Salt Rubbery Electrolytes. Macromolecules 1994, 27, 7469-7477.

(53) Cameron, G. G.; Ingram, M. D.; Harvie, J. L. Ion Transport in Polymer Electrolytes. Faraday Discuss. Chem. Soc. 1989, 88, 55-63.

(54) Stolwijk, N. A.; Heddier, C.; Reschke, M.; Wiencierz, M.; Bokeloh, J.; Wilde, G. Salt-Concentration Dependence of the Glass Transition Temperature in PEO-NaI and PEO-LiTFSI Polymer Electrolytes. Macromolecules 2013, 46, 8580-8588.

(55) Zheng, Q.; Pesko, D. M.; Savoie, B. M.; Timachova, K.; Hasan, A. L.; Smith, M. C.; Miller, T. F.; Coates, G. W.; Balsara, N. P. Optimizing Ion Transport in Polyether-Based Electrolytes for Lithium Batteries. Macromolecules 2018, 51, 2847-2858.

(56) Maginn, E. J.; Messerly, R. A.; Carlson, D. J.; Roe, D. R.; Elliott, J. R. Best Practices for Computing Transport Properties 1. SelfDiffusivity and Viscosity from Equilibrium Molecular Dynamics [Article v1.0]. Living J. Comput. Mol. Sci. 2019, 1, 1-20.

(57) Dalvi, A.; Parvathala Reddy, N.; Agarwal, S. C. The MeyerNeldel Rule and Hopping Conduction. Solid State Commun. 2012, $152,612-615$.

(58) Borodin, O.; Smith, G. D. Mechanism of Ion Transport in Amorphous Poly(Ethylene Oxide)/ LiTFSI from Molecular Dynamics Simulations. Macromolecules 2006, 39, 1620-1629.

(59) Brooks, D. J.; Merinov, B. V.; Goddard, W. A.; Kozinsky, B.; Mailoa, J. Atomistic Description of Ionic Diffusion in PEO-LiTFSI: Effect of Temperature, Molecular Weight, and Ionic Concentration. Macromolecules 2018, 51, 8987-8995.

(60) Müller-Plathe, F.; Van Gunsteren, W. F. Computer Simulation of a Polymer Electrolyte: Lithium Iodide in Amorphous Poly(Ethylene Oxide). J. Chem. Phys. 1995, 103, 4745-4756.

(61) Borodin, O.; Smith, G. D.; Douglas, R. Force Field Development and MD Simulations of Poly(Ethylene Oxide)/LiBF4 Polymer Electrolytes. J. Phys. Chem. B 2003, 107, 6824-6837.

(62) Webb, M. A.; Jung, Y.; Pesko, D. M.; Savoie, B. M.; Yamamoto, U.; Coates, G. W.; Balsara, N. P.; Wang, Z. G.; Miller, T. F. Systematic Computational and Experimental Investigation of LithiumIon Transport Mechanisms in Polyester-Based Polymer Electrolytes. ACS Cent. Sci. 2015, 1, 198-205.

(63) Mao, G.; Saboungi, M.-L.; Price, D. L.; Armand, M. B.; Howells, W. S. Structure of Liquid PEO-LiTFSI Electrolyte. Phys. Rev. Lett. 2000, 84, 5536-5539.

(64) Ebadi, M.; Eriksson, T.; Mandal, P.; Costa, L. T.; Araujo, C. M.; Mindemark, J.; Brandell, D. Restricted Ion Transport by Plasticizing Side Chains in Polycarbonate-Based Solid Electrolytes. Macromolecules 2020, 53, 764-774.

(65) Mindemark, J.; Lacey, M. J.; Bowden, T.; Brandell, D. Beyond PEO-Alternative Host Materials for Li+-Conducting Solid Polymer Electrolytes. Prog. Polym. Sci. 2018, 81, 114-143.

(66) Barteau, K. P.; Wolffs, M.; Lynd, N. A.; Fredrickson, G. H.; Kramer, E. J.; Hawker, C. J. Allyl Glycidyl Ether-Based Polymer Electrolytes for Room Temperature Lithium Batteries. Macromolecules 2013, 46, 8988-8994. 